\title{
Risk-Neutral Valuation of Participating Life Insurance Contracts in a Stochastic Interest Rate Environment *
}

\author{
Katharina Zaglauer ${ }^{\mathrm{a}}$, Daniel Bauer ${ }^{\mathrm{b}, *}$ \\ ${ }^{a}$ University of Wisconsin-Milwaukee, Department of Mathematical Sciences, 3200 \\ $N$ Cramer Street, Milwaukee, WI 53211-3029, USA \\ ${ }^{\mathrm{b}}$ Ulm University, DFG-Research Training Group 1100, Helmholtzstraße 18, 89069 \\ Ulm, Germany
}

\begin{abstract}
Over the last years the valuation of life insurance contracts using concepts from financial mathematics has become a popular research area for actuaries as well as financial economists. In particular, several methods have been proposed of how to model and price participating policies, which are characterized by an annual interest rate guarantee and some bonus distribution rules. However, despite the long terms of life insurance products, most valuation models allowing for sophisticated bonus distribution rules and the inclusion of frequently offered options assume a simple Black-Scholes setup and, in particular, deterministic or even constant interest rates.

We present a framework in which participating life insurance contracts including predominant kinds of guarantees and options can be valuated and analyzed in a stochastic interest rate environment. In particular, the different option elements can be priced and analyzed separately. We use Monte Carlo and discretization methods to derive the respective values.

The sensitivity of the contract and guarantee values with respect to multiple parameters is studied using the bonus distribution schemes as introduced in Bauer et al. (2006). Surprisingly, even though the value of the contract as a whole is only moderately affected by the stochasticity of the short rate of interest, the value of the different embedded options is altered considerably in comparison to the value under constant interest rates. Furthermore, using a simplified asset portfolio and empirical parameter estimations, we show that the proportion of stock within the insurer's asset portfolio substantially affects the value of the contract.
\end{abstract}

Key words:

participating life insurance contracts, risk-neutral valuation, stochastic interest rates, embedded options

JEL classification: G13; G22

Subj. class: IM20; IE50; IE51; IB10 


\section{Introduction}

Participating life insurance contracts are characterized by an interest rate guarantee and some bonus distribution rules, which provide the possibility for the policyholder to participate in the earnings of the insurance company. While in England or other Anglo-Saxon countries the interest rate guarantee is often given on a point-to-point basis, the predominant kinds of insurance contracts in other markets as, e.g., the German market include so-called cliquet style guarantees. Within such products, a certain guaranteed rate of return plus some surplus is credited to the policyholder's account each year. Furthermore, these contracts often contain other option features such as, for example, a surrender option.

The analysis of participating life insurance contracts with a minimum interest rate requires a realistic model of bonus payments. Grosen and Jørgensen (2000) establish some general principles for modeling bonus schemes: They argue that life insurance policies should provide a low-risk, stable and yet competitive investment opportunity. In particular, the surplus distribution should reflect the so-called "average interest principle", which states that insurers are to build up reserves in years of high returns and use the accumulated reserves to keep the surplus stable in years with low returns without jeopardizing the company's solvency. Aside from an interest rate guarantee and a distribution mechanism for excessive returns which suites these principles, the model of Grosen and Jørgensen (2000) further includes the possibility for the insured to surrender. In this case, the policyholder obtains the account value whereas the reserves remain with the company. Since the value of such a contract is pathdependent, a closed-form solution cannot be derived. Monte Carlo methods are used for the valuation of the contract.

In Jensen et al. (2001), the same valuation problem is tackled in an alternative way: Within each period, they show that the value function follows a known Partial Differential Equation (PDE), namely the Black-Scholes PDE, which can be solved using finite difference methods. At inception of each period, arbitrage arguments ensure the continuity of the value function. Based

\footnotetext{
‡ The authors thank Ralf Leidenberger and Mario Rometsch for their assistance as well as Rüdiger Kiesel, Alexander Kling, Norbert Renz, Jochen Russ, Karsten Urban, and Hans-Joachim Zwiesler for valuable comments.

* Corresponding author. Phone: +49 (731) 50 31088. Fax: +49 (731) 5031239.

Email addresses: zaglauer@uwm.edu (Katharina Zaglauer), daniel.bauer@uni-ulm.de (Daniel Bauer).
} 
on these insights, they derive a backward iteration scheme for pricing the contract. This approach is extended and generalized in Tanskanen and Lukkarinen (2004). In particular, they do not use finite differences in order to solve the PDE, but derive an integral solution which is based on the transformation of the Black-Scholes PDE into a one dimensional heat equation. Their model permits multiple distribution mechanisms including the one from Grosen and Jørgensen (2000).

The distribution mechanisms considered in Bauer et al. (2006) also satisfy the general principles provided in Grosen and Jørgensen (2000). Additionally, their general framework allows for payments to the shareholders of the company as a compensation for the adopted risk. Furthermore, Bauer et al. (2006) show how the value of a contract can be separated into its single components and derive an equilibrium condition for a fair contract.

However, all these contributions perform the valuation in a simple BlackScholes model for the financial market and, in particular, assume deterministic or even constant interest rates. Considering the long terms of insurance products, this assumption does not seem adequate. In contrast, other publications allow for a stochastic evolution of interest rates. However, these articles consider point-to-point guarantees rather than cliquet style guarantees (see Barbarin and Devolder (2005), Bernard et al. (2005), or Briys and de Varenne (1997)), or do not allow for the consideration of typical distribution schemes or option features embedded in many life insurance contracts (see, e.g., Miltersen and Persson (1999)).

The present paper fills this gap: We adopt the methodology presented in Bauer et al. (2006) and incorporate more consistent models for the behavior of interest rates into their model. In order to take into account all typical components of a participating life insurance contract, different numerical methods are presented. Besides Monte Carlo methods, we present a discretization approach based on the numerical solution of certain PDEs, which allows us to consider the non-European surrender option. We study the impact of various parameters on the contract value focusing on the parameters which emerge due to the stochasticity of the evolution of interest rates. Furthermore, using a simplified asset portfolio and empirical parameter estimations, we study the impact of the proportion of stock within the insurer's asset portfolio on the contract value.

The remainder of the paper is organized as follows: In Section 2 and 3 we briefly introduce the model and valuation methodology from Bauer et al. (2006), respectively. Section 4 presents the valuation approaches. In particular, we introduce the two stochastic interest models considered in the paper, namely the well-known models of Vasiček (1977) and Cox et al. (1985) for the evolution of the short rate, and explain our valuation algorithms as well 
as their implementation. Our results are presented in Sections 5 and 6. Besides the values of the contract and the embedded options, we examine their sensitivities toward changes in several parameters and give economic interpretations. While in Section 5 we focus on parameters that come into play due to the stochasticity of the interest rates, Section 6 is devoted to the study of the impact of the proportion of stock within the insurer's asset portfolio. Section 7 closes with a summary of the main results and an outlook for future research.

\section{Model}

We use the simplified balance sheet given in Table 2 to model the insurance company's financial situation. Here, $A_{t}$ denotes the market value of the insurer's asset portfolio at time $t, L_{t}$ the policyholders' account balance at time $t$, and $R_{t}=A_{t}-L_{t}$ the time $t$ reserve. We further assume that dividend payments $d_{t}$ to the shareholders occur at the policy anniversaries $t \in\{1, \ldots, T\}$.

Table 1

\begin{tabular}{c|c} 
Assets & Liabilities \\
\hline$A_{t}$ & $L_{t}$ \\
& $R_{t}$ \\
\hline$A_{t}$ & $A_{t}$
\end{tabular}

Simplified balance sheet

We consider only one simple life insurance contract, namely a single premium $\left(P=L_{0}\right)$ term-fix insurance contract issued at time 0 and maturing after $T$ years ignoring any charges. Given this contract, the benefit does not depend on biometric circumstances, but merely on the development of the insurer's assets and liabilities. Thus, at maturity $T$ the policyholder or the policyholder's heirs receive $P \frac{L_{T}}{L_{0}} \stackrel{P=L_{0}}{=} L_{T}$.

\subsection{Surplus distribution schemes}

The question of how the surplus is distributed to the policyholders in practice is highly delicate and demands legal as well as strategic considerations within the insurance company. Our general model allows for any management decision made at time $t$ that is dependent on the information at time $t$ only. For the numerical implementation, however, we focus on two bonus schemes from Bauer et al. (2006), who model distribution schemes based on the legal requirements (MUST-case) and the typical behavior of insurers (IS-case), respectively, within the German market. 


\section{The MUST-case}

According to the German legislation, insurance companies are obliged to grant a minimum rate of interest $g$, which is currently fixed at $2.75 \% .{ }^{1}$ Furthermore, it is required that at least a minimum participation rate $\delta=90 \%$ of the earnings on book values has to be passed on to the policyholders.

The insurers' earnings are subject to accounting rules and thus the earnings on book values are in general not equal to the earnings on market values $A_{t}^{-}-A_{t-1}^{+}$, where $A_{t}^{-}$and $A_{t}^{+}=A_{t}^{-}-d_{t}$ denote the value of the portfolio at time $t$ shortly prior to and shortly after the payment of the dividends $d_{t}$, respectively. We assume that at least a portion $y$ of the earnings on market values has to be displayed as earnings on book values in the balance sheet.

These regulations imply (see Bauer et al. (2006)) that

$$
L_{t}=(1+g) L_{t-1}+\left[\delta y\left(A_{t}^{-}-A_{t-1}^{+}\right)-g L_{t-1}\right]^{+}, t=1, \ldots, T .
$$

Assuming that the remaining portion of the earnings on book values is paid out as dividends, we obtain that

$$
\begin{aligned}
d_{t}= & (1-\delta) y\left(A_{t}^{-}-A_{t-1}^{+}\right) 1_{\left\{\delta y\left(A_{t}^{-}-A_{t-1}^{+}\right)>g L_{t-1}\right\}} \\
& +\left[y\left(A_{t}^{-}-A_{t-1}^{+}\right)-g L_{t-1}\right] 1_{\left\{\delta y\left(A_{t}^{-}-A_{t-1}^{+}\right) \leq g L_{t-1} \leq y\left(A_{t}^{-}-A_{t-1}^{+}\right)\right\}} .
\end{aligned}
$$

\section{The IS-case}

The IS-case models the typical behavior of German insurance companies regarding their bonus distribution policy over the recent years: In the past, insurance companies have tried to grant their policyholders a stable and yet competitive return. In years with high returns, asset reserves have been accumulated and utilized in years with poor returns to keep the granted rate at a stable, fairly high level. Only if the reserves dropped beneath or rose above certain levels would the companies reduce or increase the surplus, respectively. The following distribution rule originally introduced by Kling et al. (2004) was designed to model this behavior.

If crediting the target interest rate $z>g$ leads to a reserve quota between some positive constants $a$ and $b$, i.e. if

$$
a \leq x_{t} \leq b
$$

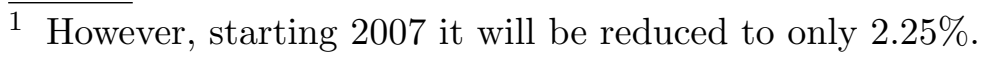


for

$$
\begin{aligned}
L_{t} & =(1+z) L_{t-1}, \\
d_{t} & =\alpha(z-g) L_{t-1}, \\
A_{t}^{+} & =A_{t}^{-}-d_{t}, \\
R_{t} & =A_{t}^{+}-L_{t},
\end{aligned}
$$

then exactly the target interest rate $z$ is credited to the policyholder. Here, $x_{t}:=\frac{R_{t}}{L_{t}}=\frac{A_{t}^{+}-L_{t}}{L_{t}}=\frac{A_{t}^{-}-d_{t}-L_{t}}{L_{t}}$ is the so-called reserve quota, and $\alpha$ is the portion of the surplus above the guaranteed level $g$ which is distributed to the shareholders. If the reserve quota falls below $a$ or rises above $b$ when crediting the target rate $z$, then the credited rate is chosen as the rate which leads to a reserve quota of exactly $a$ or $b$, respectively, where at all times the minimum participation rules from (1) and (2) need to be satisfied.

When summarizing all cases and conditions (see Bauer et al. (2006) for a more detailed description), we obtain that

$$
\begin{aligned}
L_{t}= & (1+g) L_{t-1}+\max \left\{\left[\delta y\left(A_{t}^{-}-A_{t-1}^{+}\right)-g L_{t-1}\right]^{+},\right. \\
& (z-g) L_{t-1} 1_{\left\{((1+a)(1+z)+\alpha(z-g)) L_{t-1} \leq A_{t}^{-} \leq((1+b)(1+z)+\alpha(z-g)) L_{t-1}\right\}} \\
& +\frac{1}{1+a+\alpha}\left[A_{t}^{-}-(1+g)(1+a) L_{t-1}\right] \\
& \quad 1_{\left\{(1+a)(1+g) L_{t-1}<A_{t}^{-}<((1+a)(1+z)+\alpha(z-g)) L_{t-1}\right\}} \\
& \left.+\frac{1}{1+b+\alpha}\left[A_{t}^{-}-(1+g)(1+b) L_{t-1}\right] 1_{\left\{((1+b)(1+z)+\alpha(z-g)) L_{t-1}<A_{t}^{-}\right\}}\right\},
\end{aligned}
$$

and

$$
\begin{aligned}
d_{t}= & \max \left\{\alpha\left[\delta y\left(A_{t}^{-}-A_{t-1}^{+}\right)-g L_{t-1}\right]^{+},\right. \\
& \alpha(z-g) L_{t-1} 1_{\left\{((1+a)(1+z)+\alpha(z-g)) L_{t-1} \leq A_{t}^{-} \leq((1+b)(1+z)+\alpha(z-g)) L_{t-1}\right\}} \\
& +\frac{\alpha}{1+a+\alpha}\left[A_{t}^{-}-(1+g)(1+a) L_{t-1}\right] \\
& \quad 1_{\left\{(1+a)(1+g) L_{t-1}<A_{t}^{-}<((1+a)(1+z)+\alpha(z-g)) L_{t-1}\right\}} \\
& \left.+\frac{\alpha}{1+b+\alpha}\left[A_{t}^{-}-(1+g)(1+b) L_{t-1}\right] 1_{\left\{((1+b)(1+z)+\alpha(z-g)) L_{t-1}<A_{t}^{-}\right\}}\right\} .
\end{aligned}
$$

Please note that the reserve corridor $[a, b]$ can be easily linked to the actual situation in practice: A lower bound for the bonus reserve is required for solvency reasons and an upper bound is justified by the need to stay competitive in a rising market. 


\section{Risk-Neutral Valuation}

As in Bauer et al. (2006), we assume the existence of a probability space $(\Omega, \mathcal{F}, \mathcal{Q})$ equipped with a filtration $\mathbf{F}=\left(\mathcal{F}_{t}\right)_{t \in[0, T]}$, where $\mathcal{Q}$ is a risk-neutral measure under which payment streams can be valuated as expected discounted values with respect to the numéraire process $B=\left(B_{t}\right)_{t \in[0, T]} .{ }^{2}$ The risk-neutral value of the insurance benefit (without a surrender option) is thus given by the Risk-neutral Valuation Formula (cf. Bingham and Kiesel (2004), Theorem $6.1 .4)$

$$
P^{*}=\mathbb{E}_{\mathcal{Q}}\left[B_{T}^{-1} P \frac{L_{T}}{L_{0}}\right] \stackrel{L_{0}=P}{=} \mathbb{E}_{\mathcal{Q}}\left[B_{T}^{-1} L_{T}\right]
$$

However, employing the results of the risk-neutral valuation by applying the corresponding hedging strategy so far presents difficulties within this setup: Firstly, in contrast to unit-linked products, the underlying security is not traded on the financial market, but is an asset portfolio the composition of which is influenced by the management of the insurance company. Nevertheless, it is possible to approximate the insurer's asset portfolio by a benchmark portfolio, i.e. a synthetic portfolio consisting of various instruments which are actually traded on the market. Henceforth, we will call the relevant asset portfolio the reference portfolio.

Furthermore, since the underlying is the company's asset side which is modified when the asset allocation is changed, hedging within the company's balance sheet is not possible for the insurer. To overcome this "feedback effect", we choose a different approach and assume that the insurer invests his money in the reference portfolio $A$ and leaves it there. The reference portfolio is not modified, except for the occurrence of one of the following events, which may change its value but not its composition:

(1) Dividends $d_{t}$ are paid out to the shareholders at $t=1,2, \ldots, T$; the riskneutral value at $t=0$ of these future payments is

$$
\mathrm{D}_{0}=\mathbb{E}_{\mathcal{Q}}\left[\sum_{t=1}^{T} B_{t}^{-1} d_{t}\right]
$$

(2) If the return of the reference portfolio is so poor that the interest rate guarantee cannot be fulfilled using the company's funds even if the reserve is completely dissolved, then the company needs a capital shot $c_{t}$. The

$\overline{2}$ The existence of such a risk-neutral measure implies that the financial market is arbitrage-free. 
risk-neutral value at time 0 of these capital shots is given by

$$
\mathrm{C}_{0}=\mathbb{E}_{\mathcal{Q}}\left[\sum_{t=1}^{T} B_{t}^{-1} c_{t}\right] .
$$

Thus, $\mathrm{C}_{0}$ can be interpreted as the value of the interest rate guarantee. Note that in case these capital shots are actually paid by a financial intermediator, e.g. an investment bank or a reinsurer, then, neglecting credit risk of this financial intermediator, the insurer cannot default.

Moreover, the risk-neutral value of the change of reserve is given by

$$
\Delta R_{0}=\mathbb{E}_{\mathcal{Q}}\left[B_{T}^{-1} R_{T}\right]-R_{0}
$$

where $R_{t}$ is the reserve account at time $t$.

In addition, the policyholder has the possibility to surrender his contract at any policy anniversary, say $t_{0}$, and "walk away" with the account value $L_{t_{0}}$. Using the same notation as Bauer et al. (2006), the policyholder's gain from surrendering at time $t_{0}$ is

$$
w_{t_{0}}=\max \left\{\mathrm{D}_{t_{0}}+B_{t_{0}} \mathbb{E}_{\mathcal{Q}}\left[B_{T}^{-1} R_{T} \mid \mathcal{F}_{t_{0}}\right]-R_{t_{0}}-\mathrm{C}_{t_{0}}, 0\right\}
$$

where $\mathrm{D}_{t_{0}}$ denotes the value of dividend payments in $\left[t_{0}, T\right]$ at $t_{0}$ and $\mathrm{C}_{t_{0}}$ the value of future capital shots at $t_{0}$. Hence, the value of the surrender or walk-away option at $t=0$ is given by

$$
W_{0}=\sup _{\tau \in \Upsilon_{[0, T]}} \mathbb{E}_{\mathcal{Q}}\left[B_{\tau}^{-1} w_{\tau}\right]
$$

where $\Upsilon_{[0, T]}$ denotes all stopping times with values in $\{0,1, \ldots, T\}$.

These cash-flows can be used to decompose the contract. For a "fair" contract, the values of the interest rate guarantee and the surrender option should coincide with the values of the dividend payments and the change of reserve account, i.e.

$$
\mathrm{C}_{0}+W_{0} \stackrel{!}{=} \mathrm{D}_{0}+\Delta R_{0}
$$

This equilibrium condition has the equivalent representation

$$
\begin{aligned}
P^{*}:=\mathbb{E}_{\mathcal{Q}}\left[B_{T}^{-1} P \frac{L_{T}}{L_{0}}\right]+W_{0} & =P+\mathrm{C}_{0}-\mathrm{D}_{0}-\Delta R_{0}+W_{0} \\
& \stackrel{!}{=} P .
\end{aligned}
$$

Hence, the value of the contract can be calculated in two different ways: directly as a discounted expectation or by summing up the embedded options. 
Isolating these embedded options is particularly useful for securitization purposes, since it allows for a separate valuation and thus hedging of the components of the contracts: An insurer would want to securitize the embedded options rather than the contract as a whole.

\section{Numerical Analysis}

We assume that investors can trade continuously in a frictionless, arbitragefree financial market with finite time horizon $T$ and we suppose that the single premium, which is paid at the conclusion of the contract, is invested in a well diversified reference portfolio $A$ consisting of various asset classes and evolving according to the stochastic differential equation

$$
\frac{\mathrm{d} A_{t}}{A_{t}}=r_{t} \mathrm{~d} t+\sigma_{A}\left[\rho \mathrm{d} W_{t}+\sqrt{1-\rho^{2}} \mathrm{~d} Z_{t}\right], \quad A_{0}>0 .
$$

Here $\sigma_{A}>0$ denotes the volatility of the asset process $A, \rho \in[0,1]$ characterizes the correlation between $A$ and the risk-free rate $r$, and $\left(W_{t}\right)_{t \in[0, T]}$ and $\left(Z_{t}\right)_{t \in[0, T]}$ are two independent standard Brownian motions under the equivalent risk-neutral probability measure $\mathcal{Q}$ on the complete, filtered probability space $(\Omega, \mathcal{F}, \mathcal{Q}, \mathbf{F})$, where $\mathbf{F}$ satisfies the usual conditions (cf. Karatzas and Shreve (1991), Definition 1.2.15).

The risk-free rate $r$ is assumed to evolve according to

$$
\mathrm{d} r_{t}=\kappa\left(\xi-r_{t}\right) \mathrm{d} t+\sigma_{r} r_{t}^{\gamma} \mathrm{d} W_{t}, r_{0}>0
$$

where $\kappa, \xi, \sigma_{r}$ are constants, and $\gamma \in\left\{0, \frac{1}{2}\right\}$. By choosing $\gamma=0$ we obtain the Vasiček (1977) model, and for $\gamma=\frac{1}{2}$ the Cox et al. (1985) model. ${ }^{3}$

In order for the market to be complete, we can introduce bonds as additional securities. A market without bonds would be incomplete. In particular, interest rate risk would not be hedgeable. For our considerations in this section, the market structure is not of importance, since it does not affect the valuation approach. However, it does affect the practical implementation as, under the risk-neutral measure $\mathcal{Q}$, the short rate dynamics (8) in a market with bonds in general do not coincide with the short rate dynamics in a market without bonds. Thus, the market structure has to be considered when choosing a parametrization for (8).

Taking the dividend payments into account and applying Itô's lemma, we obtain

$\overline{3}$ Note that we could easily generalize this setup by allowing for time-dependent, deterministic coefficients in (7) and (8), respectively. 


$$
A_{t}^{-}=A_{t-1}^{+} \exp \left(\int_{t-1}^{t} r_{s} \mathrm{~d} s-\frac{\sigma_{A}^{2}}{2}+\int_{t-1}^{t} \rho \sigma_{A} \mathrm{~d} W_{s}+\int_{t-1}^{t} \sqrt{1-\rho^{2}} \sigma_{A} \mathrm{~d} Z_{s}\right)
$$

and

$$
A_{t}^{+}=\max \left\{A_{t}^{-}-d_{t}, L_{t}\right\}
$$

For an Ornstein-Uhlenbeck process modeling the short rate, i.e. for $\gamma=0$, the distributions of the involved quantities are explicitly known and we can therefore implement a Monte Carlo algorithm which "exactly" simulates the corresponding quantities in order to determine the risk-neutral value of a contract without surrender option:

For the simulation we require the rate of return $r_{t}^{A}$ of the asset portfolio in each time period $[t-1, t)$ :

$$
\begin{aligned}
r_{t}^{A} & =\frac{A_{t}^{-}-A_{t-1}^{+}}{A_{t-1}^{+}} \\
& =\exp \left\{\int_{t-1}^{t} r_{s} \mathrm{~d} s-\frac{\sigma_{A}^{2}}{2}+\rho \sigma_{A} \int_{t-1}^{t} \mathrm{~d} W_{s}+\sqrt{1-\rho^{2}} \sigma_{A} \int_{t-1}^{t} \mathrm{~d} Z_{s}\right\}-1 .
\end{aligned}
$$

The normal distributions of $r_{t}$ and $\int_{t-1}^{t} r_{s} \mathrm{~d} s$ can be derived under $r_{t-1}$. Hence, $r_{t}^{A}$ can be simulated using this information, where the covariances between the respective random variables have to be taken into account. The risk-neutral value of the contract can then be approximated by

$$
L(N):=\frac{\sum_{k=1}^{N} L_{T}^{(k)} \exp \left(-\int_{0}^{T} r_{u}^{(k)} \mathrm{d} u\right)}{N}
$$

for a sufficiently large number of simulations $N$, since, by the strong law of large numbers,

$$
L(N) \stackrel{\text { a.s. }}{\longrightarrow} \mathbb{E}_{\mathcal{Q}}\left[\exp \left(-\int_{0}^{T} r_{u} \mathrm{~d} u\right) L_{T}\right](N \rightarrow \infty) .
$$

If $\gamma=\frac{1}{2}$, i.e. if the short rate is governed by a square-root process, the distribution of the integral

$$
\int_{t-1}^{t} r_{s} \mathrm{~d} s
$$

is not explicitly known and we have to discretize the integral. Thus, in our implementation, we use the relationship

$$
\begin{aligned}
r_{t+\Delta} & =e^{-\kappa \Delta}\left(r_{t}-\xi\right)+\xi+\sigma_{r} \int_{t}^{t+\Delta} e^{-\kappa(t+\Delta-s)} \sqrt{r_{s}} d W_{s} \\
& \approx e^{-\kappa \Delta}\left(r_{t}-\xi\right)+\xi+\sigma_{r} e^{-\kappa \Delta} \sqrt{r_{t}}\left(W_{t+\Delta}-W_{t}\right)
\end{aligned}
$$


where $\Delta$ is sufficiently small, and we approximate the integral by its left-hand Riemann sum. Then, the risk-neutral contract value can again be approximated by $L(N)$ from (9) for $N$ sufficiently large.

Similarly, we are able to calculate the values for the embedded options according to equations (3) and (4) from Section 3. Therefore, there are two ways to calculate the contract value: as a whole according to equation (9) or by summing up the components according to equation (6). However, for pricing the Bermuda-type surrender option, Monte Carlo methods are not preferable. Therefore, we implement a discretization approach building on the ideas of Jensen et al. (2001) and Tanskanen and Lukkarinen (2004).

\section{A Discretization Approach}

Similar to Bauer et al. (2006), we assume that the value of the policyholder's account $L_{\nu}$ at time $\nu \in\{1,2, \ldots, T\}$ is given by a known function

$$
L_{\nu}=\operatorname{Bon}_{\nu}\left(A_{\nu}^{-}, A_{\nu-1}^{+}, L_{\nu-1}\right),
$$

and analogously, that the account value adjusted by the dividend payments $d_{\nu}$ at time $\nu$ is given by a function

$$
A_{\nu}^{+}=\operatorname{Div}_{\nu}\left(A_{\nu}^{-}, A_{\nu-1}^{+}, L_{\nu-1}\right) .
$$

This leads to the following relations:

$$
\begin{gathered}
L_{t}=\left\{\begin{array}{cl}
L_{\nu-1}, t \in[\nu-1, \nu) \\
\operatorname{Bon}_{\nu}\left(A_{\nu}^{-}, A_{\nu-1}^{+}, L_{\nu-1}\right), t=\nu
\end{array},\right. \\
A_{t}^{+}=\left\{\begin{array}{cl}
A_{t}^{-}=A_{\nu-1}^{+}\left(1+\frac{A_{t}^{-}-A_{\nu-1}^{+}}{A_{\nu-1}^{+}}\right), t \in[\nu-1, \nu) \\
\operatorname{Div}_{\nu}\left(A_{\nu}^{-}, A_{\nu-1}^{+}, L_{\nu-1}\right), t=\nu
\end{array}\right.
\end{gathered}
$$

Assuming $P=L_{0}$, the fair value of the contract at maturity is equal to its payoff value, that is $V_{T}=L_{T}$, and the risk-neutral values of the European and non-European contracts at time $t$ are given by

$$
V_{t}=\left\{\begin{array}{c}
V_{t}^{\mathrm{EUR}}=\mathbb{E}_{\mathcal{Q}}\left[\exp \left(-\int_{t}^{T} r_{u} \mathrm{~d} u\right) L_{T} \mid \mathcal{F}_{t}\right] \\
V_{t}^{\mathrm{NON}-\mathrm{EUR}}=\sup _{\tau \in \Upsilon_{[t, T]}} \mathbb{E}_{\mathcal{Q}}\left[\exp \left(-\int_{t}^{\tau} r_{u} \mathrm{~d} u\right) L_{\tau} \mid \mathcal{F}_{t}\right]
\end{array}\right.
$$

respectively, where $\Upsilon_{[t, T]}$ denotes all stopping times with values in $\{\lceil t\rceil, \ldots, T\}$. 
Thus, the value of the surrender option is

$$
W_{t}:=V_{t}^{\mathrm{NON}-\mathrm{EUR}}-V_{t}^{\mathrm{EUR}} .
$$

The value $V_{t}$ of the contract at time $t$ depends on the value of the state variables $A_{t}^{-}, r_{t}, A_{\lfloor t\rfloor}^{+}$, and $L_{\lfloor t\rfloor}$ at time $t$, i.e.

$$
V_{t}=V\left(t, A_{t}^{-}, A_{\lfloor t\rfloor}^{+}, L_{\lfloor t\rfloor}, r_{t}\right) .
$$

Furthermore, it can be shown that the value function has to be almost surely continuous at $\nu \in\{1, \ldots, T\}$, i.e.

$$
V_{t} \rightarrow V_{\nu},(t \rightarrow \nu), \text { a.s. }
$$

otherwise there would be an arbitrage opportunity (see Tanskanen and Lukkarinen (2004)).

Thus, if for every $\nu$ we define the function $F_{\nu}$ for all $a, l, r$ by

$$
F_{\nu}(a, l, r):=V(\nu, a, a, l, r)
$$

for all $a, a^{\prime}, l, r$ we obtain

$$
\begin{aligned}
\lim _{t \rightarrow \nu^{-}} V\left(t, a^{\prime}, a, l, r\right) & =F_{\nu}\left(\operatorname{Div}_{\nu}\left(a^{\prime}, a, l\right), \operatorname{Bon}_{\nu}\left(a^{\prime}, a, l\right), r\right) \\
& =V\left(\nu, \operatorname{Div}_{\nu}\left(a^{\prime}, a, l\right), \operatorname{Div}_{\nu}\left(a^{\prime}, a, l\right), \operatorname{Bon}_{\nu}\left(a^{\prime}, a, l\right), r\right) .
\end{aligned}
$$

For the remainder of the section, we assume $\gamma=0$, i.e. we assume the Vasiček (1977) model for the evolution of the short rate. However, similar results can also be obtained for the Cox et al. (1985) model.

Between two policy anniversaries, the evolution of the value function $V$ only depends on changes in the asset portfolio $A$ and the interest rate $r$. Consequently, given the values of $A_{\nu-1}^{+}, L_{\nu-1}$, and given the value function at time $t_{0} \in[\nu-1, \nu)$, for $t \in\left[\nu-1, t_{0}\right]$ the value function satisfies the PDE

$$
\begin{aligned}
0= & \frac{\partial g}{\partial t}+\frac{1}{2}\left[\sigma_{A}^{2} A^{2} \frac{\partial^{2} g}{\partial A^{2}}+2 A \sigma_{A} \sigma_{r} \rho \frac{\partial^{2} g}{\partial A \partial r}+\sigma_{r}^{2} \frac{\partial^{2} g}{\partial r^{2}}\right] \\
& +r A \frac{\partial g}{\partial A}+\kappa(\xi-r) \frac{\partial g}{\partial r}-r g
\end{aligned}
$$

with final condition

$$
g\left(t_{0}, A, r\right)=V\left(t_{0}, A, A_{\nu-1}^{+}, L_{\nu-1}, r\right)
$$

which can be shown by an application of Itô's lemma. Thus, given a solution of the PDE, we can compute the value function $\forall \nu-1 \leq t<\nu$ if $V$ is known at $t=\nu$. 
Mallier and Deakin (2002) derive a closed form solution of the PDE (10) when evaluating convertible bonds. In their approach, Laplace and Mellin transformations are used to transform the PDE into an ordinary differential equation. However, we found that their approach is invalid, since the Mellin

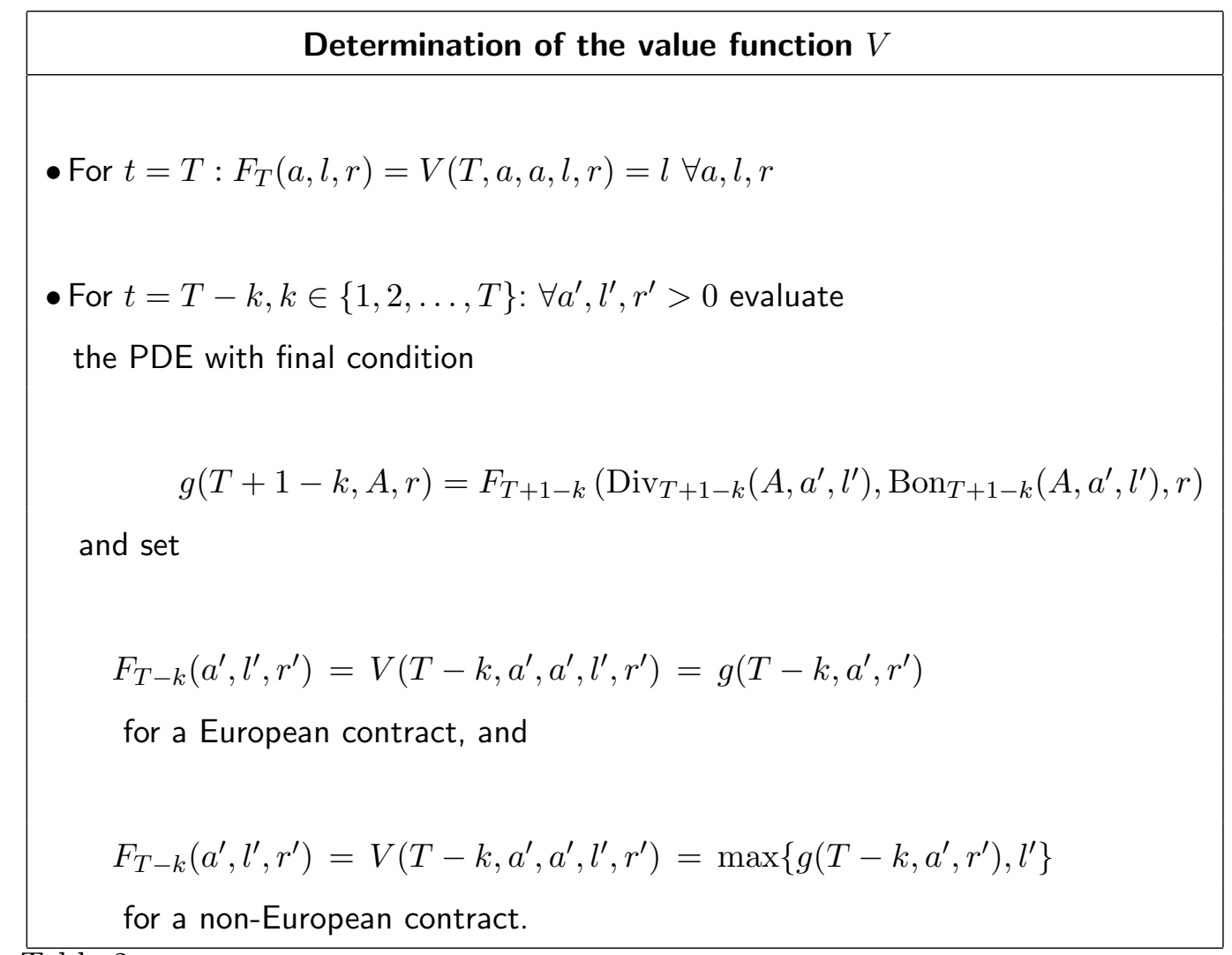

Table 2

Determination of the value function

transformation is not properly applied when transforming the PDE. Thus, we are not able to apply their ideas, but have to rely on numerical methods: By using finite differences for the approximation of first and second order space derivatives and a discrete Euler scheme for the time derivative, the solution of the PDE can be approximated. Since the value of the process $V$ is known at $t=T\left(V_{T}=L_{T}\right)$ and since it is a function of prior account values as state variables, we can obtain the value process at $t=T-1$ given the state variables at $T-1$. This leads to a backward-iterative algorithm to obtain $V_{0}$ for given state variables (see Table 2).

However, there is an infinite number of possible states. We solve this problem by using discretization methods and approximate the value function on a three-dimensional lattice:

Let $Y_{\nu} \subset \mathbb{R}_{+} \times \mathbb{R}_{+} \times \mathbb{R}$ be the set of all possible values of the state vector $y_{\nu}:=\left(A_{\nu}, L_{\nu}, r_{\nu}\right)$ and $\operatorname{Lat}_{\nu}(A, L, r) \subset Y_{\nu}$ be a finite subset of $Y_{\nu}$. $\operatorname{Lat}_{\nu}(A, L, r)$ can be different for each policy anniversary $\nu \in\{0,1, \ldots, T\}$. In order to simplify notation, we choose equidistant lattice points and use the same number 
of lattice points for each lattice $\mathrm{Lat}_{\nu}, \nu \in\{0,1, \ldots, T\}$. Thus, the lattices are determined by the marginal choices for $A, L$, and $r$ together with the number of lattice points. Let $L_{\nu}^{\min }, L_{\nu}^{\max }, A_{\nu}^{\min }, A_{\nu}^{\max }, r_{\nu}^{\min }$, and $r_{\nu}^{\max }$ denote some minimal and maximal values for $L_{\nu}, A_{\nu}$, and $r_{\nu}, \nu \in\{0,1, \ldots T\}$, and $\operatorname{Grid}_{A}, \operatorname{Grid}_{L}$, and $\operatorname{Grid}_{r}$ the number of lattice points for $A, L$, and $r$, respectively. $L_{\nu}^{\min }, L_{\nu}^{\max }, A_{\nu}^{\min }$ etc. are chosen such that the respective random variables $L_{\nu}, A_{\nu}, r_{\nu}$ remain below the maximal value and above the minimal value with a probability of more than $99 \%$. For example, we choose $A_{\nu}^{\max }$, such that

$$
\mathcal{Q}\left(A_{\nu} \leq A_{\nu}^{\max }\right) \geq 0.99
$$

for a specific à priori choice of $A_{0}$.

At maturity $T$, we have

$$
F_{T}(a, l, r)=V(T, a, a, l, r)=l \quad \forall(a, l, r) \in Y_{\nu}
$$

Thus, by numerically solving the PDE with this terminal condition, we can determine $F_{T-1}(a, l, r) \forall(a, l, r) \in \operatorname{Lat}_{T-1}(A, L, r)$. Consequently, we are given the terminal condition for the next iteration step at least on the lattice. However, for given states $a^{\prime}, l^{\prime}$ at $T-2$ and $a, r$ at $T-1$, the required values of the terminal condition

$$
g(T-1, a, r)=F_{T-1}\left(\operatorname{Div}_{T-1}\left(a, a^{\prime}, l^{\prime}\right), \operatorname{Bon}_{T-1}\left(a, a^{\prime}, l^{\prime}\right), r\right),
$$

cannot be exactly determined, since $\operatorname{Div}_{T-1}\left(a, a^{\prime}, l^{\prime}\right)$ and $\operatorname{Bon}_{T-1}\left(a, a^{\prime}, l^{\prime}\right)$ will not necessarily be located on the lattice, even if $a^{\prime}, l^{\prime}, a, r$ are on the respective lattices. Therefore, we have to interpolate between the given values of $F$ on the lattice in order to obtain $F$ for arguments between the lattice points. Since $r$ is not changed by the distribution scheme, it is sufficient to interpolate in $A$ and $L$ when only values on the lattice are required for solving the PDE. For the other policy anniversaries $t$ the computation can be carried out analogously.

With this method we can price both European and non-European contracts. The value of the surrender option can be priced as the difference of a European and a non-European contract with identical parameters. We used C++ with the FLENS and GSL-libraries for the practical implementation. ${ }^{4}$

While other valuation mechanisms similar to those in Longstaff and Schwartz (2001) could yield faster results, we consider the PDE approach as more instructive, since the corresponding PDEs include the greeks delta, gamma, and

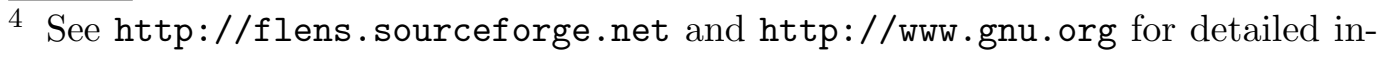
formations about the FLENS and GSL library, respectively. 
rho. ${ }^{5}$ However, for practical applications faster methods should be considered, too.

\section{Results - the Impact of Stochastic Interest Rates}

In what follows, we provide risk-neutral values of the insurance contract as well as risk-neutral values of the embedded options. We compare our results to those of Bauer et al. (2006) in order to assess how stochastic interest rates influence the respective values. Furthermore, we analyze the sensitivity of the policy value with respect to changes in the parameters that come into play due to the stochastic evolution of the interest rates.

The risk-neutral value of a life insurance policy depends on many factors and regulations. Besides the current regulatory and legal requirements, the corporate policy and the market situation affect the value of the contract. In this section, as in Bauer et al. (2006), we let the guaranteed interest rate $g=3.5 \%$, the minimum participation rate $\delta=90 \%$, the minimum portion of market value earnings that has to be displayed as book value earnings in the balance sheet $y=50 \%$, the target rate $z=5 \%$, the reserve corridor $[a, b]=[5 \%, 30 \%]$, the portion of earnings that is provided to equity holders $\alpha=5 \%$, and the volatility of the asset portfolio $\sigma_{A}=7.5 \%$. Furthermore, we set the volatility of the Ornstein-Uhlenbeck process $\sigma_{r}=1 \%$ as in Briys and de Varenne (1997) and the correlation $\rho=0.05$, which corresponds to a proportion of about $10 \%-15 \%$ of stock in the asset portfolio. ${ }^{6}$ In order to obtain comparable results for the Vasiček (1977) and the Cox et al. (1985) model, we equate the variances of the corresponding processes under $r_{0}$, which leads to a volatility parameter $\tilde{\sigma}_{r}=\sqrt{\frac{\sigma_{r}^{2}}{\xi}}$ for the square root process within the Cox et al. (1985) model. Moreover, we let the time horizon be $T=10$, the initial investment $P=10.000$, the insurer's initial reserve quota $x_{0}=10 \%$, and the initial interest rate as well as the mean reversion level $r_{0}=\xi=4 \%$ in order to be able to compare our findings to those of Bauer et al. (2006). We further choose a reversion rate $\kappa=0.14$ as in Barbarin and Devolder (2005).

As pointed out in Section 3, the risk-neutral value of a European insurance contract can be calculated in two different ways: directly, or by summation of

$\begin{array}{ll}5 & \text { For the definition and the use of the greeks, see for example Hull (2006). } \\ 6 & \text { See Section } 6 .\end{array}$ 
the individual contract components, i.e.

$$
\begin{aligned}
\text { contract value }= & \text { initial investment }(\mathrm{P}) \\
& + \text { value of the interest rate guarantee }\left(\mathrm{C}_{0}\right) \\
& - \text { change of reserve }\left(\Delta \mathrm{R}_{0}\right)-\text { value of the dividends }\left(\mathrm{D}_{0}\right) \\
& + \text { value of the surrender option }\left(\mathrm{W}_{0}\right) .
\end{aligned}
$$

The European contract value as well as its decomposition is calculated using the Monte Carlo methods introduced in Section 4. The non-European contract with walk-away option is valuated with the discretization algorithm, which was also introduced in Section 4.

\begin{tabular}{|c|r|r|r|}
\hline MUST-case & $r=4 \%$ const & OU $r_{0}=4 \%$ & CIR $r_{0}=4 \%$ \\
\hline Initial investment $\mathrm{P}$ & $10,000.00$ & $10,000.00$ & $10,000.00$ \\
\hline + Interest rate guarantee $\mathrm{C}_{0}$ & 865.9 & $1,002.7$ & $1,000.1$ \\
\hline - Value of dividends $\mathrm{D}_{0}$ & 238.1 & 242.8 & 242.2 \\
\hline - Exp. change of reserve $\Delta \mathrm{R}_{0}$ & 267.5 & 310.0 & 298.6 \\
\hline \hline Fair value European Contract & $10,360.3$ & $10,449.9$ & $10,459.3$ \\
\hline+ Value of Surrender Option $\mathrm{W}_{0}$ & 0 & 169.2 & $\mathrm{~N} / \mathrm{A}$ \\
\hline Fair value non-European Contract & $10,360.3$ & $10,619.1$ & $\mathrm{~N} / \mathrm{A}$ \\
\hline \hline IS-case & $r=4 \%$ const & OU $r_{0}=4 \%$ & $\mathbf{C I R} r_{0}=4 \%$ \\
\hline Initial investment $\mathrm{P}$ & $10,000.00$ & $10,000.00$ & $10,000.00$ \\
\hline+ Interest rate guarantee $\mathrm{C}_{0}$ & $1,052.3$ & $1,143.7$ & $1,141.4$ \\
\hline - Value of dividends $\mathrm{D}_{0}$ & 75.1 & 77.6 & 77.5 \\
\hline - Exp. Change of reserve $\Delta \mathrm{R}_{0}$ & 10.1 & 45.4 & 33.9 \\
\hline \hline Fair value European Contract & $10,967.1$ & $11,020.7$ & $11,030.0$ \\
\hline+ Value of Surrender Option $\mathrm{W}_{0}$ & 0 & 82.3 & $\mathrm{~N} / \mathrm{A}$ \\
\hline Fair value non-European Contract & $10,967.1$ & $11,103.0$ & $\mathrm{~N} / \mathrm{A}$ \\
\hline Table 3 . & & & \\
\hline
\end{tabular}

Table 3

Contract values

Table 3 displays the risk-neutral value of the contract and its components for the chosen parameter set, for a constant short rate, a short rate following an Ornstein-Uhlenbeck (OU) process (Vasiček (1977) model), and a short rate following a square root process (Cox et al. (1985) model), respectively. ${ }^{7} \mathrm{We}$ notice that for the stochastic short rate models, the contract values are higher than for a constant short rate. Furthermore, we observe that, in comparison

$\overline{7}$ For the square root process, a surrender option has not been considered. 
to the contract value itself, the interest rate option is over-proportionally influenced by the stochasticity of the short rate: Comparing the OU stochastic short rate case with the constant short rate case in the MUST-case, we observe an increase of the contract value for a European contract of approximately 90 units, but an increase of the guarantee option value of over 130 units. This deviation is compensated by a higher expected change of reserve. Moreover, we find that the surrender option is increased with stochastic interest rates: While it is never advisable to surrender given the standard parameters under constant interest rates, the surrender option becomes valuable in a stochastic interest rate environment.

Unlike the deviation between constant and stochastic interest rates, the differences between the corresponding values of the stochastic interest rate models are rather moderate. In particular, we can observe the same effects for both the Vasiček (1977) and the Cox et al. (1985) model. The same holds for our other findings if we choose the parameters in a realistic range. Therefore, in what follows, we will restrict ourselves to the Vasiček (1977) model, i.e. we focus on an $\mathrm{OU}$ process for the short rate of interest.

Regarding the sensitivity of the contract value with respect to changes in the contract parameters, the qualitative effects for the constant and stochastic interest rate environment are similar. The sensitivities with respect to parameters which are shared by the model with constant and stochastic interest rates have been studied in detail by Bauer et al. (2006). Therefore, we focus on parameters that come into play due to the stochasticity of the interest rates. Since results are very similar for European and non-European contracts, we focus on the European case where calculations are carried out using Monte Carlo methods.

\section{The Influence of the Volatilities of the Interest Rate and the Asset Process}

The volatility of the interest rate considerably affects the risk-neutral value of the insurance contract. Figure 1 shows that a change in the volatility of the OU process has a similar relative impact on the contract value in the MUST-case and in the IS-case. In both cases we observe a significant increase of the contract value: In the MUST-case, if the volatility $\sigma_{r}$ is increased from $1 \%$ to $2 \%$, the contract value increases by about 260 units, if the volatility is increased to $3 \%$, then the contract value increases by another 420 units. This strong sensitivity of the contract value is explained by the increased risk of poor returns on the money market, which makes the interest rate guarantee considerably more valuable.

Table 4 illustrates the joint influence of the parameters $\sigma_{r}$ and $\sigma_{A}$ on the contract value and the value of the interest rate guarantee. With increasing $\sigma_{A}$ and 


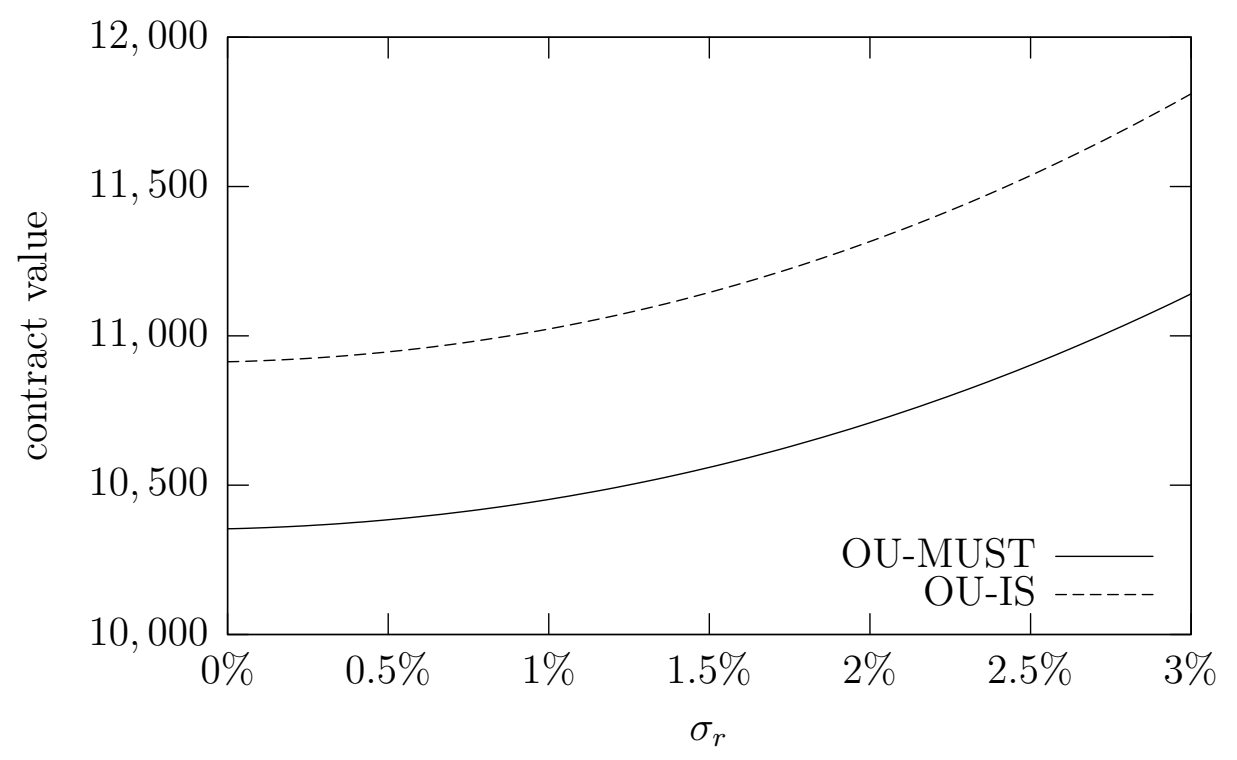

Fig. 1. Influence of $\sigma_{r}$ on the contract value

$\sigma_{r}$, the contract value increases, which can be explained by the fact that higher volatilities of asset and interest rate processes imply an increasing probability of unfavorable asset returns and of low market interest rates, respectively, which both increase the value of the guarantee option. In comparison to the contract value, the guarantee option is even increased over-proportionally as is also displayed in Table 4.

\begin{tabular}{|c|c|c|c|}
\hline OU MUST-case & $\sigma_{r}=0.5 \%, \sigma_{A}=5 \%$ & $\sigma_{r}=1 \%, \sigma_{A}=7 \%$ & $\sigma_{r}=1.5 \%, \sigma_{A}=9 \%$ \\
\hline Fair value & $9,930.6$ & $10,355.8$ & $10,849.2$ \\
\hline $\mathrm{C}_{0}$ & 351.7 & 881.8 & $1,537.2$ \\
\hline \hline OU IS-case & $\sigma_{r}=0.5 \%, \sigma_{A}=5 \%$ & $\sigma_{r}=1 \%, \sigma_{A}=7 \%$ & $\sigma_{r}=1.5 \%, \sigma_{A}=9 \%$ \\
\hline Fair value & $10,552.4$ & $10,928.2$ & $11,449.9$ \\
\hline $\mathrm{C}_{0}$ & 481.5 & $1,023.3$ & $1,679.6$ \\
\hline
\end{tabular}

Table 4

Influence of $\sigma_{r}$ and $\sigma_{A}$ on the guarantee option

We are particularly interested in parameter combinations leading to the same contract value. Besides the parameter pairs that lead to the value of a "standard contract", i.e. a contract with parameter values as introduced in the beginning of this section, the parameter combinations that lead to a "fair" contract are of interest. Here, we call a contract "fair" if the initial investment and the contract value coincide, i.e. if the equilibrium condition (5) from Section 3 is fulfilled. Figure 2 presents combinations of $\sigma_{A}$ and $\sigma_{r}$ which lead to a fair contract and to a standard contract in the MUST-case. 


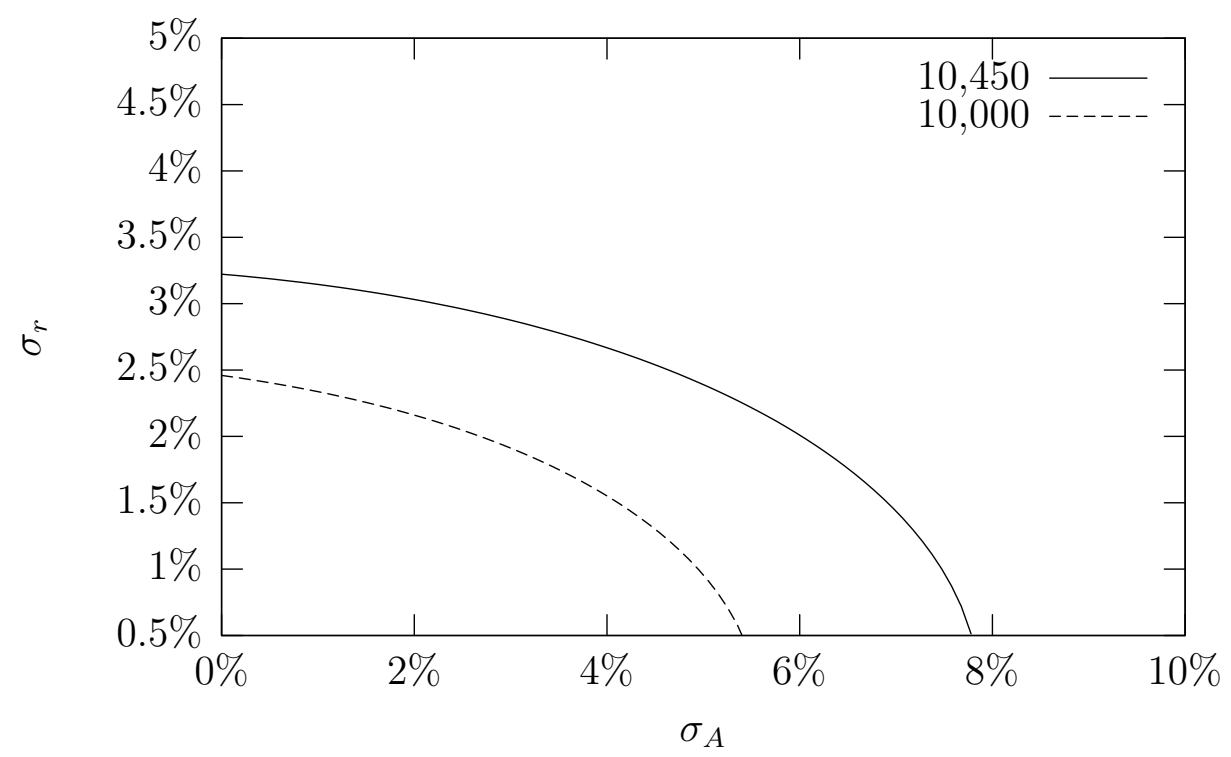

Fig. 2. Parameter combinations of $\sigma_{r}$ and $\sigma_{A}$, OU-MUST

If the volatility $\sigma_{r}$ of the interest rate process is increased, in order to keep the contract value on the same level, the volatility of the asset process has to be decreased. However, if $\sigma_{r}$ and $\sigma_{A}$ exceed $3.2 \%$ and $7.8 \%$ respectively, neither a standard contract nor a fair contract can be created. A fair contract even requires $\sigma_{r}$ and $\sigma_{A}$ to remain below $2.5 \%$ and $5.5 \%$, respectively. This indicates that in order to keep their liabilities on the same level, the insurance companies have to ensure that the asset portfolio is not too volatile, for example by mainly investing in low risk assets such as bonds and money market accounts rather than risky assets such as stock. On the other hand, the short rate must not be too volatile. Hence, if a rising volatility for the money market rates is observed, the insurance company should decrease the portion of stock in its asset portfolio in order to decrease the asset volatility $\sigma_{A}$. Conversely, in times of low volatilities on the money market, the portion of stock and other risky investments with moderate volatilities can be increased. However, even with a very low interest rate volatility or even a constant interest rate, the $7.8 \%$ bound for the volatility $\sigma_{A}$ remains. The results in the IS-case are similar.

In order to consider changes of the proportion of risky assets within the insurer's portfolio properly, we have to simultaneously adjust the correlation parameter $\rho$ in equal measure as a change in this proportion affects the composition of the asset portfolio, and thus the correlation between asset portfolio and money market. However, our analysis shows that the impact of changes of the correlation parameter is rather small, and thus the results remain more or less the same when simultaneously adjusting the correlation coefficient (see Section 6).

Instead of adjusting the portfolio composition when volatilities on the market change, the insurer can also intervene by adjusting the target rate $z$. To assess 


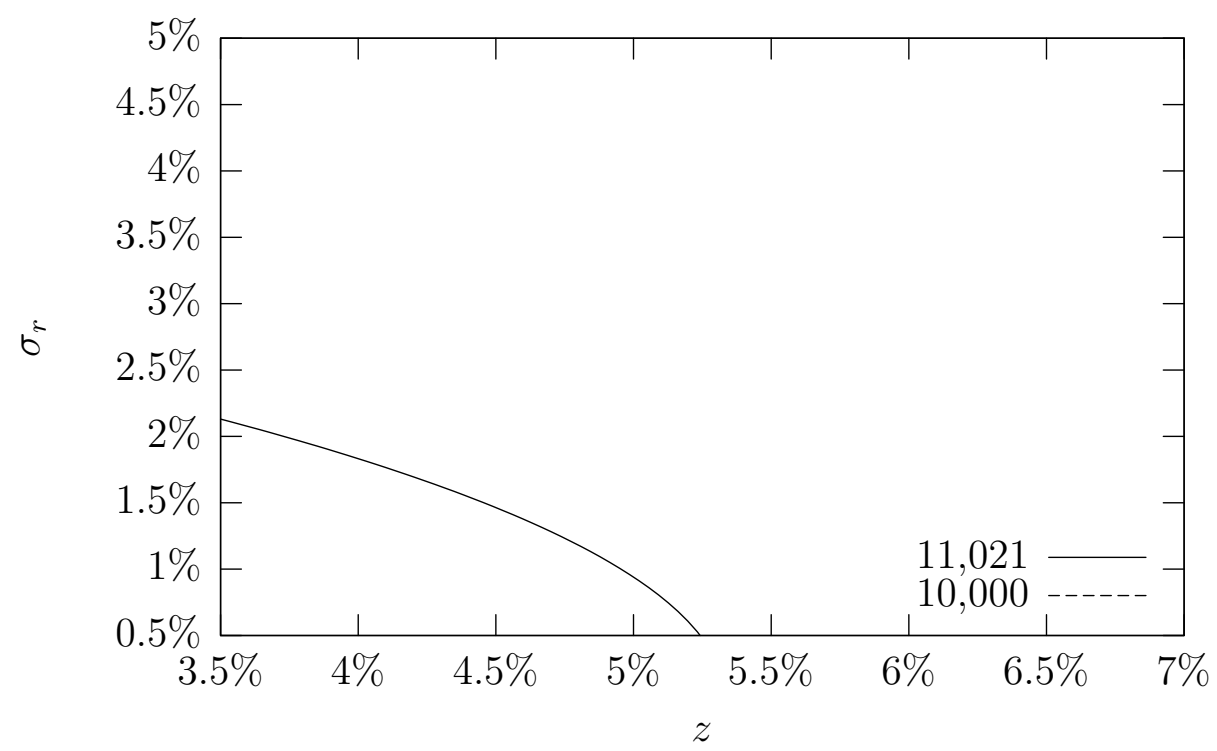

Fig. 3. Parameter combinations of $z$ and $\sigma_{r}$, OU-IS

how interest rate volatility and the target rate $z$ jointly influence the contract value, in Figure 3 we illustrate the pairs of $z$ and $\sigma_{r}$ leading to a standard contract. There are no combinations that lead to a fair contract with a value of 10,000 units. We find that if $z$ is considerably higher than $5.3 \%$, then even an interest volatility of zero cannot lead to the value of a standard contract. Furthermore, if the interest rate volatility increases from $1 \%$ to $2 \%$, a decrease of the target rate of almost $1.25 \%$ is necessary to keep the contract value at the same level. Conversely, if the interest volatility increases from $0.5 \%$ to $1.5 \%$, a decrease of the target rate of less than $0.75 \%$ is sufficient for the contract value to remain the same. This phenomenon is due to the fact that when the target rate is rather high and interest rate volatilities are rather low, the target rate poses the greatest influence on the contract value, whereas if the target rate is lower and the interest rate volatility is relatively high, the interest rate guarantee and thus the interest rate volatility have a bigger impact on the contract value.

\section{Results - the Impact of the Stock-proportion}

The impact of the stock proportion within the insurer's asset process on the contract value is connected to both, the influence of the asset volatility and the influence of the correlation between interest rate and asset. If the portfolio structure is altered, both the volatility of the portfolio and the correlation change. Thus, in order to study the impact of the stock proportion on the contract value, we need to investigate this interrelation. 
So far, we have chosen the model parameters so that it is possible to compare our results with those presented in Bauer et al. (2006), i.e. the initial interest rate as well as the mean reversion parameter $r_{0}=\xi=4 \%$ and the asset volatility $\sigma_{A}=7.5 \%$ have been chosen corresponding to the constant short rate $r$ and the asset volatility used in their contribution, respectively. For the interest rate models, we used parameterizations as proposed in the literature; however, since our underlying asset differs from specifications in other articles, no adequate value for the correlation coefficient $\rho$ can be found.

Hence, in order to obtain an idea about this correlation coefficient and in order to assess the influence of the composition of the insurer's asset portfolio properly, empirical investigations are necessary.

We assume that the insurer's assets are invested in the money market and a well diversified stock portfolio at constant proportions. Thus, the return of the reference portfolio can be determined as the weighted average of the returns of the stock portfolio and the money market rates. In our empirical analysis, we use monthly $\mathrm{DAX}^{8}$ and German call money rates for different time periods. We estimate the volatility of the reference portfolio $\sigma_{A}$ using the standard variance estimator for the normal distributed logarithms of the returns of the synthetic reference portfolio, and we use maximum likelihood estimates based on Walter (1996) for the interest rate parameters $\kappa, \xi$, and $\sigma_{r}$. Finally, we estimate $\rho$ as the correlation of the normalizations of the log returns of the reference portfolio and the call money rates, respectively, using the standard covariance estimator. The results for a stock proportion of $15 \%$ and, consequently, a money market proportion of $85 \%$ for two different time periods are given in Table 5 .

\begin{tabular}{|c|c|c|c|}
\hline & orig. parameters & estimates for 1960-2006 & estimates for 1996-2006 \\
\hline$\kappa$ & 0.14 & 0.79 & 0.19 \\
\hline$\xi$ & $4.0 \%$ & $5.0 \%$ & $2.7 \%$ \\
\hline$\sigma_{r}$ & $1.0 \%$ & $3.1 \%$ & $0.5 \%$ \\
\hline$\rho$ & 0.05 & 0.02 & 0.03 \\
\hline$\sigma_{A}$ & $7.5 \%$ & $2.9 \%$ & $3.6 \%$ \\
\hline
\end{tabular}

Table 5

Parameter estimates

Considering the comparatively high value for the volatility used in Section 5, we see that the choice of a correlation parameter of $\rho=0.05$ was adequate. We find that the other parameter estimates differ considerably from the parametrization used in Section 5. However, we use historical data for our empirical estimates and we do not consider bond prices. Thus, the interest rate parame-

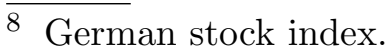


ters are not adjusted by a market price of risk. Even though, within the model, this adjustment can have an impact on the values of $\kappa$ and $\xi$ only but not on $\sigma_{r}$ or $\sigma_{A}$, it is well known that historical volatilities may well be different from implied volatilities. Thus, these deviations are not necessarily a sign that the parametrization used in Section 5 is inadequate.

Henceforth, in contrast to Section 5 where we considered a representative contract from a typical German insurer's portfolio as in Bauer et al. (2006), we now consider a just recently concluded contract with minimum interest rate $g=2.75 \%$ and $r_{0}=3 \%$ according to the current legislative regulations and the current market average for money market returns. Furthermore, since we are considering a contract with maturity $T=10$ years, we use the parameter values according to our empirical estimations based on the time period 19962006. The risk-neutral value of the insurance contract is calculated for different portions of stock and, hence, for different values of $\rho$ and $\sigma_{A}$. The data is fitted by a function $f: \mathbb{R} \rightarrow \mathbb{R}$ and the plot for the OU MUST-case is given in Figure 4.

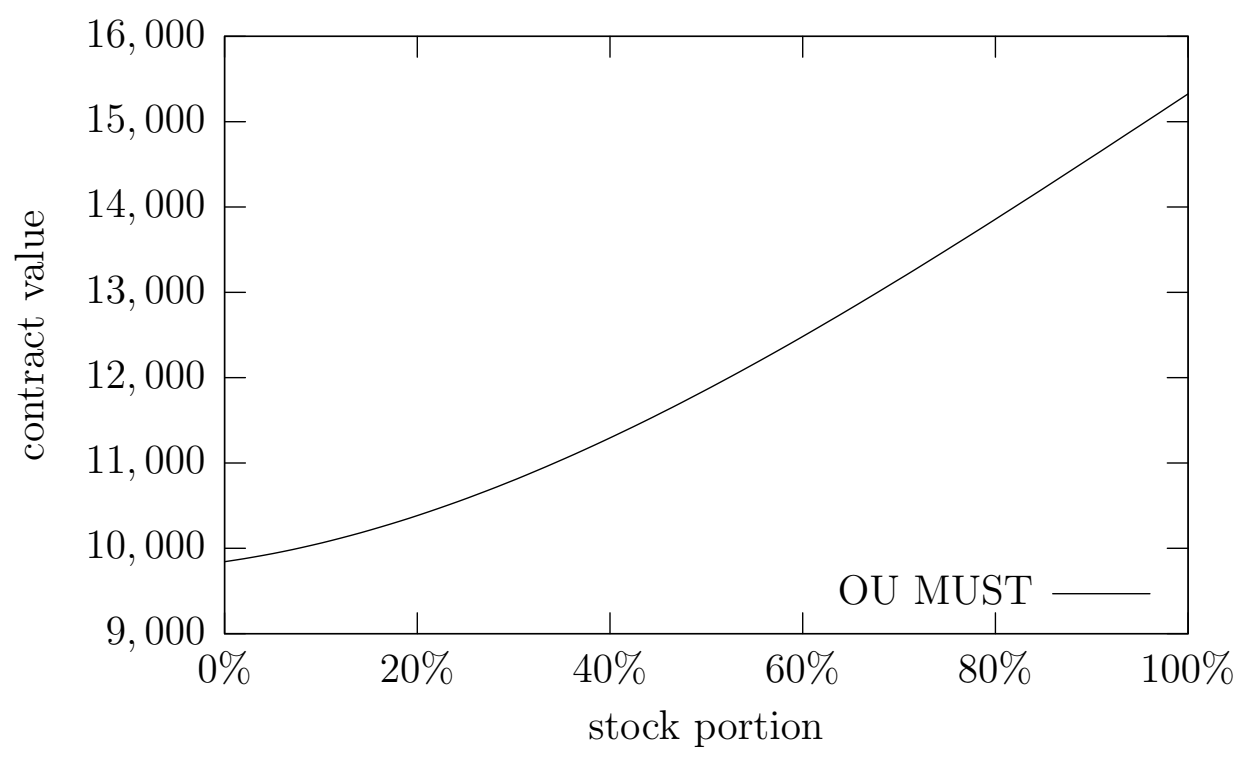

Fig. 4. Influence of the stock proportion on the contract value

We observe that with increasing stock portion within the asset portfolio, the contract value increases, which is due to the increasing risk of unfavorable asset returns represented by an increasing asset volatility $\sigma_{A}$. If the portfolio consists of $15 \%$ stock, which is realistic for a typical German insurance company, the contract value amounts to $10,157.0$ units. With a stock portion of $0 \%$, the contract is worth just a little under 10,000 units, indicating that in order to offer a fair contract in the current interest rate environment, insurers should be hardly invested in stock.

Table 6 shows how the contract value and the value of the embedded options 


\begin{tabular}{|c|r|r|r|}
\hline OU MUST-case & orig. param. & $\mathbf{1 9 6 0 - 2 0 0 6}$ & $\mathbf{1 9 9 6 - 2 0 0 6}$ \\
\hline Fair value & $10,449.9$ & $8,778.0$ & $10,157.0$ \\
\hline Interest rate guarantee $\mathrm{C}_{0}$ & $1,002.7$ & 34.9 & 233.8 \\
\hline Value of dividends $\mathrm{D}_{0}$ & 242.8 & 196.9 & 105.8 \\
\hline Exp. change of reserve $\Delta \mathrm{R}_{0}$ & 310.0 & $1,060.0$ & -29.0 \\
\hline \hline OU IS-case & orig. param. & $\mathbf{1 9 6 0 - 2 0 0 6}$ & $\mathbf{1 9 9 6 - 2 0 0 6}$ \\
\hline Fair value & $11,020.7$ & $9,809.8$ & $10,957.4$ \\
\hline Interest rate guarantee $\mathrm{C}_{0}$ & $1,143.7$ & 84.4 & 434.2 \\
\hline Value of dividends $\mathrm{D}_{0}$ & 77.6 & 88.1 & 53.4 \\
\hline Exp. change of reserve $\Delta \mathrm{R}_{0}$ & 45.4 & 186.5 & -576.6 \\
\hline
\end{tabular}

Table 6

Contract values with original parameters/estimated parameters

change if we use the estimated parameters for both periods compared with the originally used parameters, in each case for a stock proportion of $15 \%$. We find that the results vary a lot between the parameters of the different periods: The contract value differs by more than $10 \%$ for both, the MUST- and the IS-case. However, the results for both parameterizations are below the results for the original parameters used in Section 5 and those in Bauer et al. (2006). Especially the value of the interest rate guarantee is reduced considerably.

Thus, under our empirical estimates, the situation seems less alarming. However, for example in the IS-case for current parameter values, there still is a big gap between the price and the risk-neutral value of the contract, and the interest rate guarantee is still of considerable value. Thus, embedded options need to be taken into account when pricing the contracts and new risk-management approaches such as securitizing or hedging the respective guaranteed should be considered.

\section{Conclusion}

We present a valuation model for participating life insurance contracts which, in particular, allows for a stochastic evolution of interest rates. In order to focus on the basic effects, only a very simple kind of insurance contract, namely a term fix contract with a single up-front premium is considered. We present two different bonus distribution schemes for the insurance contract, which are adapted to the German regulatory framework, namely the MUST-case considering only compulsory payments due to legal and regulatory requirements, and the IS-case in which additionally corporate behaviors are taken into account. 
The life insurance contract is valuated and analyzed using methods from modern financial mathematics. However, due to the legal situation and the special features of the German insurance industry, the requirements for applying the resulting hedging strategies are not automatically fulfilled. The problem is encountered by using the cash-flow model from Bauer et al. (2006) which makes it possible to apply the concept of risk-neutral valuation and, in particular, to price and hedge the embedded options separately.

For the instantaneous short rate two different stochastic models are considered: the Vasiček (1977) model and the Cox et al. (1985) model. The OrnsteinUhlenbeck process within the Vasiček (1977) model is easier to handle, since the respective stochastic differential equation has a closed form solution. However, the process can take negative values, which may limit the applicability of the model. The Cox et al. (1985) short rate model cannot become negative under certain conditions and therefore presents a better model for the interest rate evolution. However, the Cox et al. (1985) model is more delicate to handle.

The insurance contract itself and the embedded options are complex, pathdependent derivatives. Hence, it is not possible to obtain closed form solutions for their risk-neutral values and numerical methods have to be applied. We present Monte Carlo algorithms, which allow for the valuation of a European contract as well as the embedded options, and a discretization approach which allows for the valuation of Bermuda style walk-away options in non-European contracts by solving a certain partial differential equation numerically. Besides calculating contract values, we perform sensitivity analyses with respect to those parameters that come into play due to the stochasticity of the interest rate.

It turns out that due to the additional source of uncertainty in the model, the risk-neutral value of an insurance contract with stochastic short rates always exceeds the value of a contract with a constant or deterministic short rate for a comparable parameter choice. With increasing volatility of the interest rate process, the contract value also increases. Even though the values under stochastic and constant interest rates do not differ tremendously for realistic choices of the interest rate volatility, the decomposition into the various embedded options is altered considerably. In particular, the value of the interest rate guarantee is increased over proportionally compared with the contract value.

Furthermore, we show that the composition of the insurer's asset portfolio influences the contract value considerably. Using empirical parameter estimations, we show that the contracts value for different proportions of stock within the reference portfolio between $0 \%$ and $100 \%$ is altered by more than $50 \%-$ thus, insurers should be careful in their investment decisions, since they have 
a big impact on the values of the embedded options and, thus, on the value of the contract as a whole.

Even though we observe that under the influence of stochastic short rates the value of the insurance contract as a whole exceeds the initial premium paid in most cases, our empirical studies show that this gap is highly dependent on the parametrization used. In particular, when estimating the respective values from data for different time periods, the results differ tremendously. The incorporation of stochastic interest rates is important, since market interest rates do not remain constant over the long lifetimes of insurance contracts. However, it is very difficult to choose an adequate model and, within a given interest rate model, to calibrate the parameters adequately.

Empirical studies further show that the distribution of the log returns of the asset process differ from the assumed normal distribution. Thus, it could be worthwhile to consider other processes to model the asset portfolio. We could further extend the model by considering an asset portfolio consisting of several different asset classes such as bonds, real estate etc. instead of a single asset process the composition of which is described via correlations. In addition, in order to obtain a more applicable model, it would be interesting to determine hedging strategies for the insurance contract and for the embedded options.

\section{References}

Bauer, D., Kiesel, R., Kling, A., Ruß, J., 2006. Risk-Neutral Valuation of Participating Life Insurance Contracts. Insurance: Mathematics and Economics, 39: 171-183.

Barbarin, J., Devolder, P., 2005. Risk measure and fair valuation of an investment guarantee in life insurance. Insurance: Mathematics and Economics, 37: 297-323.

Bernard, C., Le Courtois, O., Quittard-Pinon, F., 2005. Market Value of Life Insurance Contracts under Stochastic Interest Rates and Default Risk. Insurance: Mathematics and Economics, 36: 499-516.

Bingham, N.H., Kiesel, R., 2004. Risk-Neutral Valuation. 2nd ed., Springer Finance, Springer, London.

Briys, E., de Varenne, F., 1997. On the Risk of Insurance Liabilities: Debunking some Common Pitfalls. The Journal of Risk and Insurance, 64: 673-694.

Cox, J.C., Ingersoll, J.E., Ross, S.A., 1985. A Theory of the Term Structure of Interest Rates. Econometrica, 53:385-407.

Jensen, B., Jørgensen, P.L., Grosen, A., 2001. A Finite Difference Approach to the Valuation of Path Dependent Life Insurance Liabilities. Geneva Papers on Risk and Insurance Theory, 26: 57-84.

Grosen, A., Jørgensen, P.L., 2000. Fair Valuation of Life Insurance Liablities: 
The Impact of Interest Guarantees, Surrender Options, and Bonus Policies. Insurance: Mathematics and Economics, 26: 37-57.

Hull, J.C., 2006. Options, Futures, and Other Derivatives. 6th ed., PrenticeHall, Englewood Cliffs, New Jersey.

Karatzas I., Shreve, S.E., 1991. Brownian Motion and Stochastic Calculus. 2nd ed., Graduate Texts in Mathematics 113, Springer, New York.

Kling, A., Richter, A., Ruß, J., 2004. The Interaction of Guarantees, Surplus Distribution, and Asset Allocation in With Profit Life Insurance Policies. To appear in Insurance: Mathematics and Economics.

Longstaff, F.A., Schwartz, E.S., 2001. Valuing American Options by Simulation: A Simple Least-Squares Approach. Review of Financial Studies, 14: $113-147$.

Mallier, R. and Deakin, A.S., 2002. A Green's Function for a Convertible Bond using the Vasicek Model. Journal of Applied Mathematics, 5: 219-232.

Miltersen, K.R., Persson, S.A., 1999. Pricing Rate of Return Guarantees in a Heath-Jarrow-Morton Framework. Insurance: Mathematics and Economics, 25: 307-325.

Walter, U., 1996. Die Bewertung von Zinsoptionen. Gabler Verlag, Wiesbaden, Germany.

Tanskanen, A.J., Lukkarinen, J., 2004. Fair Valuation of Path-Dependent Participating Life Insurance Contracts. Insurance: Mathematics and Economics, 33: 595-609.

Vasiček, O., 1977. An Equilibrium Characterization of the Term Structure. Journal of Financial Economics, 5: 177-188. 